\title{
Emprego de Altas Doses de Amiodarona Via Oral na Reversão da Fibrilação Atrial no Pós-Operatório de Cirurgia Cardíaca
}

\author{
João Carlos Vieira da Costa Guaragna, Valério Martins, Tatiana Marlowe Cunha Brunini, \\ José Luis Linhatti, Fábio Branco de Araújo Brauner, Renan Canibal Pires, Luiz Carlos Bodanese
}

Porto Alegre, RS

Objetivo - Relatar a experiência no emprego de altas doses de amiodarona via oral $(1800 \mathrm{mg} / \mathrm{d})$ na reversão da fibrilação atrial (FA) em pacientes submetidos à cirurgia cardíaca.

Métodos - Analisados, retrospectivamente, 80 pacientes que apresentaram FA no pós operatório de cirurgia cardíaca, constituindo 2 grupos: grupo A com 28 pacientes em uso de amiodarona e grupo $\mathrm{B}$ recebendo digital, sendo que este grupo foi subdividido no grupo $\mathrm{C}$ com 21 pacientes onde foi associada amiodarona, quando não houvesse reversão da arritmia em $48 \mathrm{~h}$. As diferenças foram consideradas significativas para um valor de $\mathrm{P}<0,05$.

Resultados-AFA esteve presente em $19,4 \%$ dos pacientes operados, com predomínio no sexo masculino e entre 60-69 anos. No grupo A, a reversão ocorreu em 78,6\% dos casos, grupo B, o digital obteve sucesso em $60 \%$ das vezes e no grupo C, a taxa de sucesso foi de $90 \%$.

Conclusão - O uso de altas doses de amiodarona via oral, isolada ou em combinação com digital pode ser segura e eficaz no tratamento da FA em pós operatório de cirurgia cardíaca.

Palavras-chave: amiodarona, fibrilação atrial, cirurgia cardíaca

\section{High Dose Amiodarone for the Reversion of Atrial Fibrillation during the Postoperative Period of Cardiac Surgery}

Purpose - To report our experience using high dose oral amiodarone $(1,800 \mathrm{mg} /$ day $)$ for the reversion of atrial fibrillation to sinus rhythm in patients submitted to cardiac surgery.

Methods - We retrospectively analyzed the records of 80 patients who had atrial fibrillation during the postoperative period after cardiac surgery, initially divided in two groups: group A, 28 patients that used amiodarone, and group B composed of patients receiving digoxin. The latter group was divided further in a third group (C), with $21 \mathrm{pa}-$ tients in which amiodarone was associated with digoxin if there was no reversion of the arrhythmia after 48 hours of treatment. The observed differences were considered significant at $\mathrm{P}<0.05$.

Results - Atrial fibrillation occurred in $19.4 \%$ of the patients submitted to surgery, predominating in males, 60 to 69 years-old. In group A there was reversion to sinus rhythm in $78.6 \%$ of the cases. In group B digoxin succeeded in $60 \%$, and in group C $90 \%$ of the patients reverted to sinus rhythm.

Conclusion - High dose oral amiodarone, alone or combined to digoxin, can be safe and effective for the treatment of atrial fibrillation after cardiac surgery.

Key-words: amiodarone, atrial fibrillation, cardiac surgery

Arq Bras Cardiol, volume 69 (nº 6), 401-405, 1997

A fibrilação atrial (FA) é uma arritmia comum no pósoperatório (PO) de cirurgia cardíaca com uma incidência em torno de $60 \%$ dos casos $^{1-3}$.

Hospital São Lucas - Faculdade de Medicina da Pontifícia Universidade Católica do Rio Grande do Sul

Correspondência: João Carlos V. Costa Guaragna - Av. Ipiranga, 6690 - conj. 320 - 90610-000 - Porto Alegre, RS

Recebido para publicação em 6/1/97

Aceito em 8/10/97
Os fatores associados com o desenvolvimento da FA no PO de cirurgia cardíaca são diferentes dos ocorridos em outras situações clínicas. McAlister e $\mathrm{col}^{3}$ consideram que a etiologia é multifatorial, estando envolvidos tempo de circulação extracorpórea, níveis de catecolaminas circulantes, pericardite, anemia, trauma cirúrgico, drogas anestésicas, distúrbios metabólicos e retirada de betabloqueadores.

Existem diferentes propostas de tratamento da FA aguda mas, na situação específica do PO de cirurgia cardíaca, poucos trabalhos foram publicados ${ }^{1-4}$. O emprego de 
drogas, como quinidina, digital e amiodarona endovenosa (EV) mostrou-se eficaz às custas de muitos efeitos colaterais. Por isso muitos esforços têm sido realizados para se encontrar uma dose de amiodarona via oral (VO) que tenha rapidez de ação semelhante à $\mathrm{EV}$, mas com menos efeitos adversos ${ }^{5}$.

O objetivo deste artigo é relatar a experiência do nosso serviço no uso de altas doses de amiodarona VO, na reversão da FA, em pacientes submetidos à cirurgia cardíaca. Os resultados foram comparados com o uso isolado do digital e sua associação à amiodarona.

\section{Métodos}

De fevereiro/94 a abril/96 foi feito estudo observacional, retrospectivo e descritivo onde foram selecionados, entre 537 casos que realizaram cirurgia cardíaca no Hospital São Lucas da PUCRS, 80 pacientes do seu serviço de PO que apresentaram FA sem repercussão hemodinâmica, na fase intra-hospitalar, após a alta da UTI, divididos, inicialmente, em dois grupos: grupo A com 28 pacientes usando amiodarona e grupo B com 52 pacientes recebendo apenas digital, sendo que este grupo foi subdividido em um grupo C com 21 pacientes, onde foi associada amiodarona, caso não houvesse reversão da arritmia nas primeiras $48 \mathrm{~h}$ (fig. 1).

A dose utilizada de amiodarona foi de $1800 \mathrm{mg}$ VO por

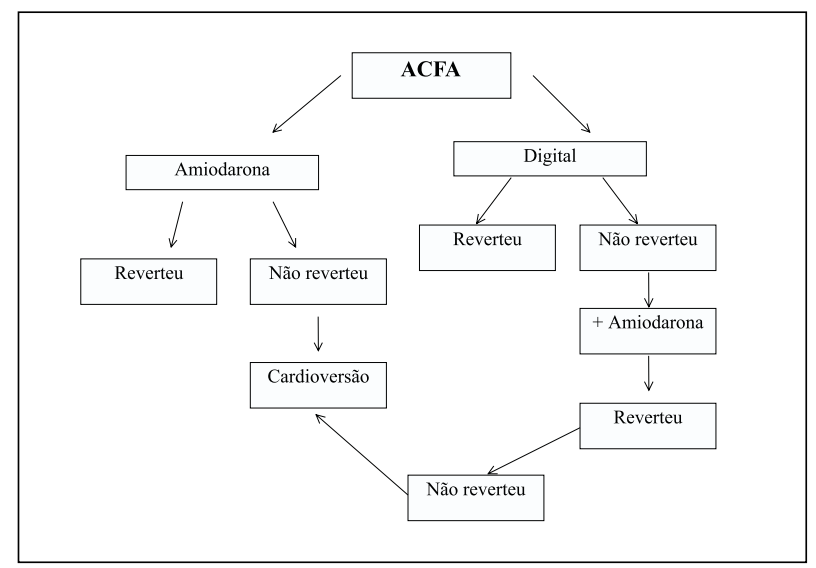

Fig. 1 - Algoritmo para o tratamento da fibrilação atrial no pós-operatório de cirurgia cardíaca. ACFA- arritmia completa por fibrilação atrial.

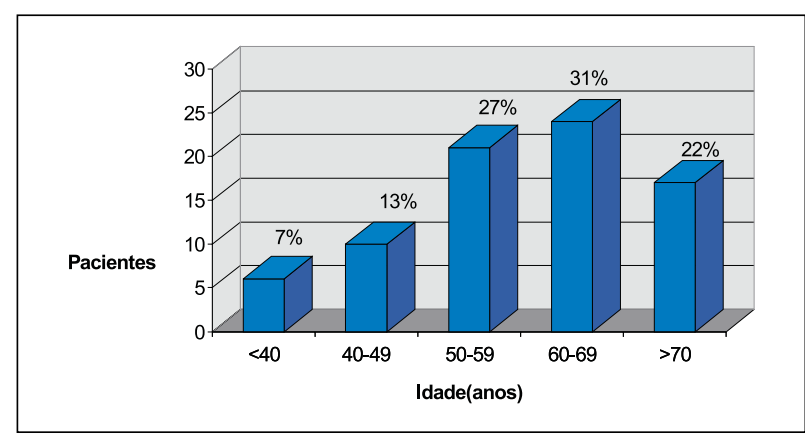

Fig. 2 - Prevalência da fibrilação atrial no pós-operatório de cirurgia cardíaca de acordo com a faixa etária $(\mathrm{n}=80)$.

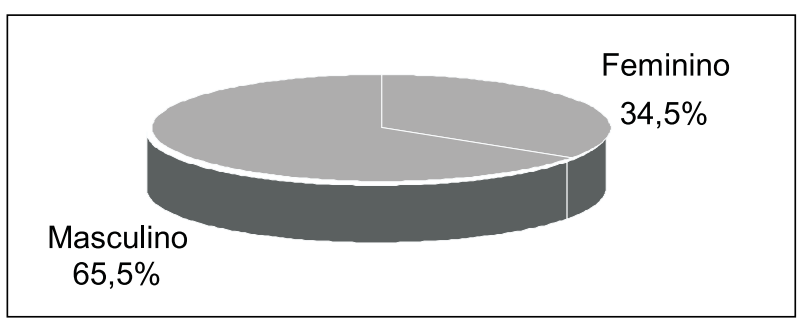

Fig. 3 - Prevalência da fibrilação atrial no pós-operatório de cirurgia cardíaca em relação ao sexo.

dia, divididos em três tomadas (8/8h). Caso não ocorresse sucesso, a dose era repetida por mais 24 ou $48 \mathrm{~h}$, totalizando um máximo de $72 \mathrm{~h}$ de terapêutica, isto é, $5400 \mathrm{mg}$.

Para a digitalização foi utilizado lanatosídeo $\mathrm{C}$ na dose de $0,4 \mathrm{mg}$ a $0,8 \mathrm{mg}$ EV/dia, considerada padrão na literatura ${ }^{4}$.

A função ventricular foi avaliada pela ventriculografia radioisotópica e definida como subnormal quando a fração de ejeção (FE) era $<50 \%$. Os pacientes do grupo A, possuíam FE mais elevada que os outros dois grupos $(\mathrm{P}<0,05)$.

Observamos, também, que no grupo $\mathrm{A}$ os pacientes eram mais jovens quando comparados aos do grupo B e C $(\mathrm{P}<0,05)$.

A reversão da FA foi avaliada pela realização de eletrocardiograma, diariamente em todos os pacientes, além do exame clínico.

\begin{tabular}{|c|c|c|c|c|}
\hline \multicolumn{5}{|c|}{ Tabela I - Características dos pacientes } \\
\hline \multicolumn{2}{|l|}{ Grupos } & $\begin{array}{c}\text { A } \\
\text { Amiodarona } \\
(n=28)\end{array}$ & $\begin{array}{c}\text { B } \\
\text { Digital } \\
(n=52)\end{array}$ & $\begin{array}{c}\text { C } \\
\text { Digital }+ \\
\text { Amiodarona } \\
(n=21)\end{array}$ \\
\hline \multicolumn{2}{|c|}{ Idade média, anos } & $55(12)$ & $60(10)$ & $61(8)$ \\
\hline \multicolumn{2}{|c|}{$\begin{array}{c}\text { Feminino/masculino } \\
(\%)\end{array}$} & $\begin{array}{c}10 / 18 \\
(36) /(64)\end{array}$ & $\begin{array}{c}15 / 37 \\
(29) /(71)\end{array}$ & $\begin{array}{c}5 / 16 \\
(24) /(76)\end{array}$ \\
\hline $\begin{array}{l}\text { Tipo cirurgia ( } \\
\text { 1- RM } \\
\text { 2- Troca valvar }\end{array}$ & & $\begin{array}{l}14(50) \\
15(50)\end{array}$ & $\begin{array}{l}38(73) \\
14(27)\end{array}$ & $\begin{array}{c}15(71) \\
6(29)\end{array}$ \\
\hline $\mathrm{N}^{\circ}$ de vasos & $\begin{array}{l}2 \\
3\end{array}$ & $\begin{array}{l}7 \\
7\end{array}$ & $\begin{array}{l}12 \\
26\end{array}$ & $\begin{array}{l}6 \\
9\end{array}$ \\
\hline \multicolumn{2}{|l|}{$\mathrm{CEC}, \min (\mathrm{DP})$} & $87(29)$ & $91(32)$ & $87(31)$ \\
\hline \multicolumn{2}{|l|}{ FEJ, \% (DP) } & $55(18)$ & $44(16)$ & $46(13)$ \\
\hline \multirow[t]{4}{*}{ Angina classes } & I & 1 & - & - \\
\hline & II & 4 & 13 & 6 \\
\hline & III & 4 & 5 & 2 \\
\hline & IV & 5 & 14 & 5 \\
\hline \multicolumn{5}{|l|}{ ICC classes } \\
\hline & I & 2 & 2 & - \\
\hline & II & 6 & 7 & 3 \\
\hline & III & 3 & 7 & 1 \\
\hline & IV & 2 & - & - \\
\hline $\begin{array}{l}\text { RM - revascular } \\
\text { de ejeção; ICC- }\end{array}$ & . & $\begin{array}{l}\text { cárdica; CEC } \\
\text { cia cardíaca }\end{array}$ & $\begin{array}{l}\text { dlação extra } \\
\text { stiva }\end{array}$ & ea; FE- fração \\
\hline
\end{tabular}




\begin{tabular}{|lcccc|}
\hline \multicolumn{5}{|c|}{ Tabela II - Resultados } \\
\hline Grupos & $\begin{array}{c}\text { A } \\
\text { Amiodarona } \\
(\mathrm{n}=28)\end{array}$ & $\begin{array}{c}\text { B } \\
\text { Digital } \\
(\mathrm{n}=52)\end{array}$ & $\begin{array}{c}\text { C } \\
\text { Digital + } \\
\text { amiodarona } \\
(\mathrm{n}=21)\end{array}$ \\
\hline Reversão (\%) & $22(78,6)$ & $31(60)$ & $19(90)$ \\
Falência (\%) & $6(21,4)$ & $21 \quad(40)$ & $2 \quad(10)$ \\
Tempo de reversão & & & & \\
(em dias) & 1 & 11 & 28 & 16 \\
& 2 & 7 & 3 & 1 \\
& 3 & 4 & - & 2 \\
& 3 & 2 & - & - \\
\hline
\end{tabular}

\section{Resultados}

A prevalência da FA foi de $19,4 \%$ no PO. A distribuição conforme a faixa etária é apresentada na figura 2 . Houve predomínio do sexo masculino (fig. 3).

No grupo A houve reversão da FA a ritmo sinusal em 22 $(78,6 \%)$ pacientes sendo que em $82 \%$ deles a reversão ocorreu nas primeiras $48 \mathrm{~h}$, sendo a reversão em $24 \mathrm{~h}$, de $50 \%$.

Para o grupo B 31 (60\%) resolveram a arritmia, sendo $90 \%$ deles nas primeiras $24 \mathrm{~h}$ de tratamento.

Já no grupo C 19 (90\%) obtiveram sucesso, sendo $84 \%$ também nas primeiras $24 \mathrm{~h}$ após o início da associação medicamentosa.

O sucesso terapêutico dos grupos A e $\mathrm{C}$ foi superior ao do grupo $\mathrm{B}(\mathrm{P}<0,05)$.

A eficácia entre o grupo A e o grupo $\mathrm{C}$, quando houve associação com digital, foi a mesma do ponto de vista estatístico.

Os efeitos colaterais foram pouco freqüentes para todos os grupos, sendo que nenhum deles ocasionou repercussão clínica significativa (tab. III). Intoxicação digitálica aconteceu em dois pacientes do grupo C. O diagnóstico foi estabelecido pela presença de vômitos e bloqueio atrioventricular de $2^{\circ}$ grau.

Obervou-se, também, que os pacientes que utilizaram inicialmente o digital apresentavam FE mais baixa, possuíam maior número de vasos comprometidos e eram mais sintomáticos, tornando a associação de amiodarona eficaz na reversão da arritmia, não acarretando prejuízos clínicos para os mesmos.

\begin{tabular}{|lccc|}
\hline \multicolumn{4}{|c|}{ Tabela III - Efeitos colaterais } \\
\hline Grupos & $\begin{array}{c}\text { A } \\
\text { Amiodarona } \\
(\mathrm{n}=28)\end{array}$ & $\begin{array}{c}\mathrm{B} \\
\text { Digital } \\
(\mathrm{n}=52)\end{array}$ & $\begin{array}{c}\mathrm{C} \\
\text { Digital + } \\
\text { amiodarona } \\
(\mathrm{n}=21)\end{array}$ \\
\hline Diarréia & - & 1 & - \\
Vômitos & 1 & 1 & - \\
BAV $1^{\circ}$ grau & 1 & 1 & - \\
Intoxicação & - & - & 2 \\
Digitálica & - & & \\
\hline BAV- bloqueio atrioventricular. & & \\
\hline
\end{tabular}

\section{Discussão}

AFA no PO de cirurgia cardíaca é uma arritmia de grande prevalência, sendo que muitos pacientes permanecem assintomáticos, não necessitando retornar a UTI para reversão imediata. A maioria dos estudos registra, como maior ocorrência da FA, os $2^{\circ}$ e $3^{\circ}$ dias do $\mathrm{PO}^{6-9}$. Nossos pacientes apresentaram FA após a alta da UTI.

Muitos autores tratam esta arritmia apenas quando desencadeia instabilidade hemodinâmica, considerando-a auto-limitada ${ }^{1,2,4}$. Em nosso serviço, optamos pelo tratamento pois a FA pode reduzir o débito cardíaco em até $30 \%$, levando à instabilidade hemodinâmica. Além disto, aumenta o consumo de $\mathrm{O}_{2}$, acarretando em prejuízo, principalmente, nos pacientes com revascularização incompleta, podendo tornar-se fonte de êmbolos, sendo a principal causa de tromboembolismo e ictus cerebral ${ }^{1,9,10}$.

McAlister e $\operatorname{col}^{3}$ demonstraram maior eficácia da quinidina em relação à amiodarona $\mathrm{EV}$, porém com alta taxa de efeitos colaterais. Comparou-se, também, o uso de digoxina e amiodarona EV no tratamento das arritmias supraventriculares no PO de cirurgia cardíaca, concluindo os autores que ambas as drogas são seguras e eficazes ${ }^{1}$.

As propriedades farmacocinéticas da amiodarona são complexas e não totalmente compreendidas. Após a administração VO da droga a absorção é lenta e variável com uma biodisponibilidade oscilando entre 22 e $86 \%{ }^{5,11-13}$. É altamente lipofílica ocasionando sua larga distribuição, sendo acumulada extensivamente em todos os tecidos, prolongando o início do seu efeito.

Em seres humanos concentrações mais altas foram encontradas no fígado, gordura e pulmões ${ }^{11-13}$. A concentração miocárdica de amiodarona é superior àquela do plasma. Após a absorção da amiodarona, a relação da concentração plasmática e miocárdica aumenta com o passar do tempo, atingindo um platô após 60 a 90min, período no qual as concentrações plasmáticas já caem para níveis muito baixos, momento em que a concentração miocárdica de amiodarona é cerca de $10 \mathrm{a} 50$ vezes à do plasma ${ }^{13,14}$.

Os dados experimentais sobre o acúmulo tecidual correspondem à observação clínica de que efeitos antiarrítmicos são retardados após o início do tratamento com amiodarona (latência terapêutica) ${ }^{14}$. Uma maneira potencial de reduzir esse retardo consiste em usar altas doses iniciais de impregnação nos primeiros dias de tratamento ${ }^{15,16}$. Baseados nessas constatações é que optamos por empregar altas doses de amiodarona VO.

Foi demonstrado que o tempo necessário para se obter os efeitos antiarrítmicos era encurtado, quando da administração de $1400 \mathrm{mg} / \mathrm{dia}$, em comparação com $200 \mathrm{mg} / \mathrm{dia}^{17}$. Alguns autores verificaram que, com a administração oral, as concentrações plasmáticas da amiodarona eram mais altas a cada dia de tratamento ${ }^{18}$. Foi proposto um modelo farmacocinético de três compartimentos com o conceito de que os efeitos antiarrítmicos exigem o acúmulo do medicamento num compartimento periférico ${ }^{5}$. Assim, é provável que elevadas doses de amiodarona por VO consigam manter con- 
centrações, igualmente elevadas na circulação, reduzindo sua rápida captação tecidual. Este efeito dependeria da biodisponibilidade oral individual que é extremamente variável.

Recentemente foram estudados os efeitos hemodinâmicos de grandes doses de amiodarona VO. Há um efeito vasodilatador inicial, seguido por um decréscimo na freqüência cardíaca com elevação na pressão de enchimento ventricular e decréscimo no índice cardíaco ${ }^{19}$. Em nosso trabalho, os pacientes em que utilizamos primeiramente digital, possuíam FE mais baixa. Entretanto, quando acrescentamos amiodarona, o sucesso terapêutico foi alcançado sem o surgimento de alterações hemodinâmicas, como efeito colateral.

Do ponto de vista clínico, a amiodarona é droga com eficácia comprovada nas arritmias cardíacas. A experiência mundial salienta seu uso por via $\mathrm{EV}$, com vários efeitos colaterais relacionados em seu emprego, sendo a instabilidade hemodinâmica o mais temido ${ }^{3,20}$.

No início desta década foi publicado estudo sobre o emprego de amiodarona em altas doses VO $(800 \mathrm{mg} /$ dia durante 4 dias), em 19 pacientes com taquiarritmia atrial refratária e alcançada uma taxa de sucesso, isto é, conversão a ritmo sinusal em $48 \mathrm{~h}$, em $63 \%$ dos pacientes. Efeitos colaterais sem repercussão clínica foram relatados em $42 \%$ dos casos. Os autores concluíram que a amiodarona em altas doses VO é droga eficaz para rápida conversão de taquiarritmias supraventriculares em ritmo sinusal ${ }^{15}$.

Adrivet e col ${ }^{16}$ relataram sua experiência com amiodarona VO em dose única elevada, $25-30 \mathrm{mg} / \mathrm{kg}$, na reversão de taquiarritmias atriais. No mesmo trabalho apresentaram os resultados com amiodarona EV. Concluíram pela eficácia da amiodarona para cardioversão química das taquiarritmias e sugeriram que a VO em doses elevadas é adequada para a maioria dos pacientes sendo que a via $\mathrm{EV}$ deve ser utilizada em poucos casos ${ }^{16}$. Um estudo retrospectivo sobre o uso da amiodarona EV e, após VO, para FA em PO de revascularização miocárdica relatou taxa de sucesso nas primeiras $48 \mathrm{~h}$ de $93,4 \%{ }^{2}$.
Recentemente Martin e col ${ }^{21}$, na Argentina, relataram o uso de amiodarona em altas doses pare reverter FA no PO de cirurgia cardíaca. Estudaram 35 pacientes, sendo que 15 receberam amiodarona isolada e 20 após o insucesso com digital e quinidina. Os pacientes receberam uma dose de ataque EV, em 2h, de 300-450mg de amiodarona seguida de uma dose de manutenção de $900-1050 \mathrm{mg} / 24 \mathrm{~h} \mathrm{EV}$ durante dois dias, seguida de $400 \mathrm{mg} /$ dia VO. A taxa de sucesso encontrada por esses autores foi de $97 \%$ com a grande maioria dos pacientes tendo a arritmia revertida nas primeiras $24 \mathrm{~h}$. A recidiva ocorreu em $12 \%$, todos tendo seu ritmo restaurado pela dose $\mathrm{VO}^{21}$.

Nosso trabalho apresenta dados semelhantes, contudo, empregamos como dose de ataque VO pelo menos $50 \%$ maior, o que está acima dos valores médios preconizados. Entretanto, como mostra a tabela III a incidência de efeitos colaterais foi pequena e os mesmos não acarretaram efeitos hemodinâmicos importantes. Salientamos, também, que o fato de o paciente fazer uso de três comprimidos de $8 / 8 \mathrm{~h}$ não trouxe alterações gastrointestinais, para os nossos pacientes, pois apenas dois apresentaram vômitos e diarréia, não relacionados à intoxicação digitálica (tab. III).

Houve, também, um alto índice de reversão com a associação digital + amiodarona (90\%). Deve ser enfatizado, porém, que esse regime favorece o risco de intoxicação digitálica.

Nosso estudo apresenta, como limitação, o fato de não ser randomizado, sem grupo controle e retrospectivo, não permitindo, portanto, conclusões definitivas. Entretanto, nossos dados sugerem que a amiodarona, em altas doses VO (1800mg/dia), pode ser útil na reversão de FA que ocorre no PO de cirurgia cardíaca quando usada isoladamente e sugere, também, que naqueles pacientes que não obtiveram sucesso com o emprego do digital, a associação de amiodarona em altas doses VO foi eficaz e segura, não acarretando prejuízos hemodinâmicos. Consideramos importante a realização de novos estudos prospectivos, randomizados e com placebo, o que poderia demonstrar, por exemplo, a incidência de reversão espontânea.

\section{Referências}

1. Lauer MS, Eagle KA, Buckley MJ, Dessanctis WR - Atrial fibrillation following coronary artery bypass surgery. Prog Cardiovasc Dis 1989; 5: 367-78.

2. Contini GA, Astorri E, Cavozza Cet al - Short term amiodarone for atrial fibrillation after coronary surgery. Cuore 1993; X: 195-203.

3. McAlister HF, Luke RA, Whitelock RM, Smith WM - Intravenous amiodarone bolus versus oral quinidine for atrial flutter and fibrillation after cardiac operations. J Thorac Cardiovasc Surg 1990; 99: 91-8.

4. Waldo AL, Henthorn RW, Epstein AE et al - Diagnosis and treatment of arrhythmia during and following open heart surgery. Med Clin North Am 1984; 68: 1153-70.

5. Siddoway LA, McAllister CB, Wilkinson GR et al - Amiodarone dosing. A proposal based on its pharmokinetics. Am Heart J 1983; 106: 951-6.

6. Reed GL, Singer DE, Picard EH et al - Stroke following coronary - artery bypass surgery: A case control estimate of the risk from carotid bruits. NEng J Med 1988; 319: 246-50.

7. Fuller JA, Adams GG, Buxton AE - Atrial fibrillation after coronary artery bypass grafting. J Thorac Cardiovasc Surg 1990; 98: 821-5.
8. Yousif H, Davies G, Oakley CM - Peri-operative supraventricular arrythmias in coronary surgery. Int J Cardiol 1990; 26: 313-18.

9. Piegas LS, Gun G - Fibrilação atrial no pós-operatório de revascularização miocárdica. Rev Soc Cardiol ESP 1994; 4: 305-11.

10. Wolf PA, Kannel WB, McGEE DL et al - Duration of atrial fibrillation and imminence of stroke: The Framingham Study. Stroke 1983, 14: 664-7.

11. Singh BN - Amiodarone: Pharmacological, electrophysiological and clinical profile of an sinusal antiarrhytmic compound. In: Singh BN, Wellnes HJJ, Hiraoka $\mathrm{H}$, eds - Electropharmacological Control of Cardiac Arrhythmia. Mount KisCo, NY: Futura Publishing, 1994: 497-522.

12. Singh BM, Sarma JSM - Amiodarone and amiodarone derivations. In: Singh BM, Dzam VJ, Vanhoutte PM, Woosley RL, eds - Cardiovascular Pharmacology and Therapeutics. New York: Churchill Livingstone 1994; 689-709.

13. Heger JJ, Psytowsky EM, Miles WH, Zipe DP - Uso clínico e farmacologia da amiodarona. Clin Med Am Norte 1984; 1425-51.

14. Kerr, Rosenbaum MD, Chiale PA - Amiodarone. In: Messerly FH, ed -Cardiovascular Drug Therapy. Philadelphia: WB Saunders Co 1996: 1247-64. 
15. Mostow ND, Vrobel TR, Noon D, Rakita - Rapid control of refractory atrial tachyarrhytmias with high-dose oral amiodarone. Am Heart J 1990; 120: 135663.

16. Andrivet P, Boubakri E, Dove JP, Mach V, Ngoc CV - A clinical study of amiodarone as a single oral dose in patients with recent - onset atrial tachyarrhytmia. Eur Heart J 1994; 15: 1396-402.

17. Rakita L, Sobol SM - Amiodarone in the treatment of refractory ventricular arrhythmia. Importance and safety of initial high-dose therapy. JAMA 1983; 250: 1293-5.

18. Heger JJ, Prystowsky EN, Zipes DP - Relationship between amiodarone dosage, drug concentrations and adverse side effects. Am Heart J 1983; 106: 931-5.

19. Gottlieb SS, Riggio OW, Lauria S et al - High dose oral amiodarone loading exerts important hemodynamic actions in patients with congestive heart failure. J Am Coll Cardiol 1994; 23: 560-4

20. Kopleman HA, HorowitzLN-Efficacy and toxicity of amiodarone for the treatment of supra ventricular tachyarrythmias. Prog Cardiovasc Dis 1989; 5: 355-66.

21. Martin O, Eduardo G, José C et al - Intravenous amiodarone in coronary artery bypass surgery complicated with atrial fibrillation. VI World Congress of Cardiac Rehabilitation. Buenos Aires, 1996. 